\title{
On the Ampleness of Positive CR Line Bundles over Levi-flat Manifolds
}

\author{
by
}

\author{
Masanori ADACHI
}

\begin{abstract}
We give an example of a compact Levi-flat CR 3-manifold with a positive-along-leaves CR line bundle which is not ample with respect to transversely infinitely differentiable CR sections. This example shows that we cannot improve the regularity of the Kodaira type embedding theorem for compact Levi-flat CR manifolds obtained by Ohsawa and Sibony.
\end{abstract}

2010 Mathematics Subject Classification: Primary 32V30; Secondary 32E10, 32V25, $53 \mathrm{D} 35$.

Keywords: projective embedding, Levi-flat CR manifold, holomorphic disc bundle, pseudoconvexity, confoliation.

\section{$\S 1$. Introduction}

We are going to study function theory on Levi-flat CR 3-manifolds, i.e., 3-manifolds foliated by Riemann surfaces. If we view them as families of Riemann surfaces, we can expect analogy with classical theory of Riemann surfaces, such as the Riemann-Roch theorem. But there is a new ingredient: dynamics of the foliation. We should face a subtle interaction between complexity of Levi foliations and existence of CR functions; and especially in the case where the Levi-flat CR 3-manifold is realized as a real hypersurface in a complex surface, it should be reflected in pseudoconvexity of the complement and complex geometry of the ambient space. There are several attempts in this direction. We refer the reader to the works of Inaba [12] and Barrett [1]

We investigate such a phenomenon in a problem relating to an analogue of Kodaira's embedding theorem. Ohsawa and Sibony proved the following Kodaira type embedding theorem.

Communicated by K. Ono. Received December 11, 2012.

M. Adachi: Graduate School of Mathematics, Nagoya University, Furo-cho, Chikusa-ku, Nagoya 464-8602, Japan;

e-mail: m08002z@math.nagoya-u.ac.jp

(C) 2014 Research Institute for Mathematical Sciences, Kyoto University. All rights reserved. 
Theorem ([17, Theorem 3], refined in [15]). Let $M$ be a compact $\mathcal{C}^{\infty}$ Levi-flat $C R$ manifold equipped with a $\mathcal{C}^{\infty} C R$ line bundle $L$. Suppose $L$ is positive along leaves, i.e., there exists a $\mathcal{C}^{\infty}$ hermitian metric on $L$ such that the restriction of the curvature form to each leaf is everywhere positive definite. Then, for any $\kappa \in \mathbb{N}, L$ is $\mathcal{C}^{\kappa}$-ample, i.e., there exists $n_{0} \in \mathbb{N}$ such that one can find CR sections $s_{0}, \ldots, s_{N}$ of $L^{\otimes n}$, of class $\mathcal{C}^{\kappa}$, for any $n \geq n_{0}$, such that the ratio $\left(s_{0}: \cdots: s_{N}\right)$ embeds $M$ into $\mathbb{C P}^{N}$.

We can make the regularity $\kappa \in \mathbb{N}$ arbitrarily large, although we need to take $n_{0}$ sufficiently large. A natural question is whether we can improve the regularity to $\kappa=\infty$. The answer is no, in general, as the following case-study tells us.

Main Theorem. Let $\Sigma$ be a compact Riemann surface, and $\mathcal{D}$ a holomorphic disc bundle over $\Sigma$. Denote its associated compact $\mathcal{C}^{\infty}$ Levi-flat CR manifold by $M=\partial \mathcal{D}$ in the associated flat ruled surface $\pi: X \rightarrow \Sigma$. Take a positive line bundle $L$ over $\Sigma$. Suppose that $\mathcal{D}$ has a unique non- \pm holomorphic harmonic section $h$ with $\operatorname{rank}_{\mathbb{R}} d h=2$ on an open dense set. Then $\pi^{*} L \mid M$ is positive along leaves, but never $\mathcal{C}^{\infty}$ ample.

We can easily see that the pull-back bundle $\pi^{*} L \mid M$ is positive along leaves. Thus, this theorem states non- $\mathcal{C}^{\infty}$-ampleness of such $\mathrm{CR}$ line bundles.

The assumption is fulfilled for the following explicit example (Example 3.2): Let $\Sigma$ be a compact Riemann surface of genus $\geq 2$. Fix an identification of a universal covering $\widetilde{\Sigma} \simeq \mathbb{D}$ and regard $\pi_{1}(\Sigma) \simeq \Gamma<\operatorname{Aut}(\mathbb{D})$ as a Fuchsian representation of $\Sigma$. Take a non-trivial quasiconformal deformation of $\Gamma$, say $\rho: \Gamma \rightarrow \operatorname{Aut}(\mathbb{D})$. Set $\mathcal{D}:=\widetilde{\Sigma} \times \mathbb{D} /(z, \zeta) \sim(\gamma z, \rho(\gamma) \zeta)$ for $\gamma \in \Gamma$.

Another research direction related to the analogue of Kodaira's embedding theorem is the problem of projective embedding of compact laminations; we can find a similar phenomenon in the work of Fornæss and Wold [8, Theorem 5.1] where they study compact $\mathcal{C}^{1}$ hyperbolic laminations. We also refer the reader to related works by Gromov [10, pp. 401-402], Ghys [9, §7], Deroin [4] and Martínez Torres [13].

The organization of this paper is as follows. In $\S 2$, we introduce basic notions pertaining to Levi-flat CR manifolds. In $\S 3$, we recall and refine a classification result for holomorphic disc bundles with an emphasis on Takeuchi 1completeness of certain holomorphic disc bundles. This notion is also known as $\log \delta$-pseudoconvexity [2] or strong Oka property [11], and is of interest from the viewpoint of confoliation. In $\S 4$, we state a Bochner-Hartogs type extension theorem for CR sections. We give a self-contained proof for the reader's convenience. In $\S 5$, we prove the Main Theorem and pose some further questions. 


\section{§2. Levi-flat CR manifolds}

Let us recall basic notions briefly. For simplicity, we assume that all manifolds and bundles are at least $\mathcal{C}^{\infty}$-smooth.

\section{$\S 2.1$. In terms of foliation}

A $\mathcal{C}^{\infty}$ Levi-flat $C R$ manifold is a triple $(M, \mathcal{F}, J)$ where $M$ is a $\mathcal{C}^{\infty}$ manifold, $\mathcal{F}$ is a $\mathcal{C}^{\infty}$ foliation on $M$ of real codimension one, and $J$ is a $\mathcal{C}^{\infty}$ section of $\operatorname{End}(T \mathcal{F})$ that induces a complex structure on each leaf, i.e., $J^{2}=-\mathrm{Id}$ and the set of smooth sections of $T^{1,0}:=\operatorname{Ker}(J-i \mathrm{Id}) \subset \mathbb{C} \otimes T \mathcal{F} \subset \mathbb{C} \otimes T M$ is closed under the Lie bracket. The foliation $\mathcal{F}$ is referred to as the Levi foliation of the Levi-flat CR manifold $M$.

The simplest example, which provides the local structure of Levi-flat CR manifolds, is $M=\mathbb{C}^{n-1} \times \mathbb{R}$ where $\mathcal{F}$ is given by its leaves $\left\{\mathbb{C}^{n-1} \times\{t\}\right\}_{t \in \mathbb{R}}$ and $J$ is induced from the standard complex structure of $\mathbb{C}^{n-1}$. Any $\mathcal{C}^{\infty}$ Levi-flat $\mathrm{CR}$ manifold can be constructed by gluing some open subsets of $\mathbb{C}^{n-1} \times \mathbb{R}$ together using leafwise holomorphic $\mathcal{C}^{\infty}$ maps.

We say a function $f: M \rightarrow \mathbb{C}$ is a $C R$ function if it is leafwise holomorphic.

\section{§2.2. In terms of CR geometry}

We will investigate Levi-flat CR manifolds in complex manifolds. The terminology of CR geometry is suitable for this purpose.

A $C R$ manifold (of hypersurface type) is a pair $\left(M, T^{1,0}\right)$ where $M$ is a $\mathcal{C}^{\infty}$ manifold of dimension $2 n-1$, and $T^{1,0}$ is a subbundle of $\mathbb{C} \otimes T M$ of $\mathbb{C}$-rank $n-1$ that satisfies $T^{1,0} \cap \overline{T^{1,0}}=0$ and the set of smooth sections of $T^{1,0}$ is closed under the Lie bracket. This notion models a real hypersurface $M$ in an $n$-dimensional complex manifold $\left(X, J_{X}\right)$; for such $M$ we can put $T^{1,0}:=T^{1,0} X \cap \mathbb{C} T M \simeq$ (the maximal $J_{X}$-invariant subspace of $\left.T M\right)$. Moreover, if the real hypersurface $M$ is given by a $\mathcal{C}^{\infty}$ defining function $r$, i.e., $r: M \subset U \rightarrow \mathbb{R}$ with $M=\{z \in U \mid r(z)=0\}$ and $d r \neq 0$ on $M$, we have $T^{1,0}=\operatorname{Ker} \partial r \subset T^{1,0} X$.

We can redefine a $\mathcal{C}^{\infty}$ Levi-flat $C R$ manifold as a $\mathcal{C}^{\infty} \mathrm{CR}$ manifold $\left(M, T^{1,0}\right)$ such that the set of smooth sections of $T^{1,0}+\overline{T^{1,0}}$ is closed under the Lie bracket. The Levi foliation $\mathcal{F}$ is recovered by integrating the distribution $\left(T^{1,0}+\overline{T^{1,0}}\right) \cap T M$. In the case that $M$ is located in a complex manifold $X$ with defining function $r$, $M$ is Levi-flat if and only if the Levi form $i \partial \bar{\partial} r \mid T^{1,0}$ is 0 as a quadratic form. This is the classical definition of Levi-flat real hypersurface.

We say a function $f: M \rightarrow \mathbb{C}$ is a $C R$ function if it is annihilated by all vectors of $T^{0,1}:=\overline{T^{1,0}}$. If $M=\{r=0\} \subset X$ and $f$ is $\mathcal{C}^{1}$, this is equivalent to saying that $\bar{\partial} \tilde{f}$ is proportional to $\bar{\partial} r$ on $M$ where $\tilde{f}$ is any $\mathcal{C}^{1}$ extension of $f$ onto a neighborhood of $M$. In particular, the restriction of any holomorphic function 
defined near $M$ is CR. In the Levi-flat case, this definition agrees with the one given before.

Remark 2.1. Only a few examples are known of compact Levi-flat real hypersurfaces. For instance, we cannot find such a compact real hypersurface in $\mathbb{C}^{n}(n \geq 2)$ since there is a strictly plurisubharmonic function $\sum_{i=1}^{n}\left|z_{i}\right|^{2}$, whose restriction to each Levi leaf gives a strictly subharmonic function, and the maximum principle forbids this. The same reasoning implies that no compact Levi-flat real hypersurface exists in Stein manifolds. A famous still open conjecture is the non-existence of compact $\mathcal{C}^{\infty}$ Levi-flat real hypersurfaces in $\mathbb{C P}^{2}$.

\section{$\S 2.3$. CR line bundles}

Let $L$ be a $\mathcal{C}^{\infty} C R$ line bundle over a Levi-flat $\mathrm{CR}$ manifold $M$, that is, a $\mathcal{C}^{\infty}$ $\mathbb{C}$-vector bundle of $\mathbb{C}$-rank 1 that possesses a trivialization cover whose transition functions are CR. Let $h$ be a $\mathcal{C}^{\infty}$ hermitian metric on $L$. We can find a connection on $L$ that is equal to the Chern connection on $(L \mid N, h)$ along any leaf $N$. We denote by $\Theta_{h}$ the curvature 2-form of the restricted connection along $T \mathcal{F}$, that is, $\Theta_{h}=-\partial_{z} \bar{\partial}_{z} \log h(z, t)$ where $(z, t): M \supset U \rightarrow \mathbb{C}^{n-1} \times \mathbb{R}$ is any foliated chart. We say $L$ is positive along leaves if there exists a hermitian metric $h$ on $L$ such that $i \Theta_{h}(\zeta, \bar{\zeta})>0$ for any non-zero $\zeta \in T^{1,0}$. If $M$ is three-dimensional, the existence of a positive-along-leaves $\mathrm{CR}$ line bundle over $M$ is equivalent to the tautness of its Levi foliation (cf. [13, Lemma 1]).

\section{§3. Holomorphic disc bundles in flat ruled surfaces}

We recall a classification result for holomorphic disc bundles, which are associated with a standard example of Levi-flat CR 3-manifolds, and supplement some results about pseudoconvexity of these spaces.

\section{§3.1. Holomorphic disc bundles}

We begin by recalling a construction of holomorphic disc bundles. Let $\Sigma$ be a compact Riemann surface. A holomorphic fiber bundle over $\Sigma$ with fiber $\mathbb{D}:=$ $\{\zeta \in \mathbb{C}|| \zeta \mid<1\}$ is called a holomorphic disc bundle over $\Sigma$. It can be easily seen that holomorphic trivializations form a flat trivializing cover, i.e., all of the transition functions are locally constant.

Hence, any holomorphic disc bundle $\mathcal{D}$ can be obtained by the suspension construction: we can find a group homomorphism $\rho: \pi_{1}(\Sigma) \rightarrow \operatorname{Aut}(\mathbb{D})$, called a holonomy homomorphism, giving a bundle isomorphism

$$
\mathcal{D} \simeq \Sigma \times_{\rho} \mathbb{D}:=\widetilde{\Sigma} \times \mathbb{D} /(z, \zeta) \sim(\gamma z, \rho(\gamma) \zeta) \quad \text { for } \gamma \in \pi_{1}(\Sigma)
$$

where $\widetilde{\Sigma}$ is a universal covering of $\Sigma$. We denote this disc bundle by $\mathcal{D}_{\rho}$. 
The group Aut $(\mathbb{D})$ of biholomorphisms of $\mathbb{D}$ consists of all Möbius transformations preserving $\mathbb{D}$, acting on the Riemann sphere $\mathbb{C P}^{1}$ and fixing the unit circle $\partial \mathbb{D}$. Thus, a holomorphic disc bundle is canonically embedded in its associated flat ruled surface, say $\pi: X_{\rho}:=\Sigma \times_{\rho} \mathbb{C P}^{1} \rightarrow \Sigma$, and the boundary of $\mathcal{D}_{\rho}$ in $X_{\rho}$, a flat circle bundle, becomes a compact $\mathcal{C}^{\omega}$ Levi-flat CR 3-manifold, say $M_{\rho}:=\Sigma \times_{\rho} \partial \mathbb{D}$. Note that $\mathcal{D}_{\rho} \rightarrow X \backslash \overline{\mathcal{D}_{\rho}},(z, \zeta) \mapsto(z, 1 / \bar{\zeta})$, is an anti-biholomorphism, which we call conjugation.

\section{§3.2. Classification}

Now we state a classification result for holomorphic disc bundles by means of harmonic sections, which is based on the works of Diederich and Ohsawa [5], [6].

Theorem 3.1. Let $\mathcal{D}$ be a holomorphic disc bundle over a compact Riemann surface $\Sigma$ and $M$ its associated Levi-flat CR 3-manifold. Then one of the following cases occurs:

(i) $\mathcal{D}$ admits a unique non-holomorphic harmonic section $h$ with $\operatorname{rank}_{\mathbb{R}} d h=2$ on an open dense set.

(ii) $\mathcal{D}$ admits a unique locally non-constant holomorphic section.

(iii) $\mathcal{D}$ admits a unique harmonic section $h$ with $\operatorname{rank}_{\mathbb{R}} d h=1$ on an open dense set.

(iv) $M$ admits one or two locally constant section(s).

(v) $\mathcal{D}$ admits a locally constant section.

Here a section is said to be harmonic if it can be lifted to a $\rho$-equivariant harmonic map $\tilde{h}: \widetilde{\Sigma} \rightarrow \mathbb{D}$ where $\mathbb{D}$ is equipped with the Poincaré metric; and a section is said to be locally constant if it is locally constant in the (flat) trivializing coordinates.

Proof of Theorem 3.1. By [5, Theorem 2], there exists either a harmonic section $h$ of $\mathcal{D}$, or a locally constant section of $M$. In the latter case, by examining possible holonomy homomorphisms, we find that there are at most two locally constant sections, which is case (iv) (cf. [6, Proposition 1.1]).

Now we suppose the former case, the existence of $h$. By applying a theorem of Sampson [18, Theorem 3] to the lift of $h$, we find that $\operatorname{rank}_{\mathbb{R}} d h$ is constant on an open dense subset of $\Sigma$. If the rank is zero or one, we have case (v) or (iii) respectively. The remaining case, where the rank is two, is classified into (ii) or (i) depending on whether $h$ is holomorphic or not.

Example 3.2. We describe examples of each case in terms of the holonomy homomorphism. 
(i) Let $\Sigma$ be of genus $\geq 2$. Fix an identification $\widetilde{\Sigma} \simeq \mathbb{D}$ and regard $\pi_{1}(\Sigma) \simeq$ $\Gamma<\operatorname{Aut}(\mathbb{D})$ as a Fuchsian representation of $\Sigma$. Take a non-trivial quasiconformal deformation of $\Gamma$, say $\rho: \Gamma \rightarrow \operatorname{Aut}(\mathbb{D})$. Then $\mathcal{D}_{\rho}$ is as in case (i). The unique harmonic section corresponds to the graph of the unique harmonic diffeomorphism $\Sigma=\mathbb{D} / \Gamma \rightarrow \mathbb{D} / \rho(\Gamma)$.

(ii) Let $\Sigma$ and $\Gamma$ be as above, and $\rho=\operatorname{Id}: \Gamma \rightarrow \Gamma \subset \operatorname{Aut}(\mathbb{D})$. Then $\mathcal{D}_{\rho}$ is as in case (ii). Its associated holomorphic section is obtained by the quotient of the diagonal set $\Delta \subset \widetilde{\Sigma} \times \mathbb{D}=\mathbb{D} \times \mathbb{D}$.

(iii) Let $\rho$ be a homomorphism from $\pi_{1}(\Sigma)$ to a subgroup of Aut $(\mathbb{D})$ generated by hyperbolic elements with a common axis and an elliptic element of order two which reverses the axis. Then $\mathcal{D}_{\rho}$ is as in case (iii). The image of the unique harmonic section corresponds to the axis.

(iv) Let $\rho$ be a homomorphism from $\pi_{1}(\Sigma)$ to an abelian subgroup of Aut $(\mathbb{D})$ that consists of all parabolic (resp. hyperbolic) elements with common fixed point(s) on $\partial \mathbb{D}$. Then $\mathcal{D}_{\rho}$ is as in case (iv). The locally constant section(s) correspond(s) to the suspension of the fixed point(s).

(v) Let $\rho$ be a homomorphism from $\pi_{1}(\Sigma)$ to an abelian subgroup of Aut $(\mathbb{D})$ that consists of elliptic elements with a common fixed point in $\mathbb{D}$, which is just isomorphic to the group of rotations $\mathrm{U}(1)$. Then $\mathcal{D}_{\rho}$ is as in case $(\mathrm{v})$. The suspension of the fixed point gives a locally constant section.

Note that for cases (iii)-(v), the descriptions above characterize the cases, respectively.

\section{§3.3. Pseudoconvexity}

Dynamical complexity of Levi foliations of flat circle bundles is directly encoded in their holonomy homomorphisms. On the other hand, it is indirectly reflected in pseudoconvexity of the holomorphic disc bundles bounded by the flat circle bundles.

Known facts on the pseudoconvexity of a holomorphic disc bundle, say $\mathcal{D}$, over a compact Riemann surface are summarized as follows:

- In all cases, $\mathcal{D}$ is weakly 1 -complete ([5, Theorem 1$])$.

- In cases (i)-(iv), $\mathcal{D}$ is 1-convex; it is in particular Stein if and only if (i), (iii) or (iv) holds ([1, Theorem 2]).

- In cases (i) and (ii), $\mathcal{D}$ is Takeuchi 1-convex ([6, Proposition 1.6 $\left.]^{1}\right)$.

We will give a supplementary result for case (i) using the following notion.

\footnotetext{
${ }^{1}$ Its proof seems to contain some errors.
} 
Definition 3.3 (Takeuchi $q$-complete space). Let $X$ be a complex manifold of dimension $n$, and $D$ a relatively compact domain in $X$ with $\mathcal{C}^{2}$ boundary. Then $D$ is said to be Takeuchi q-complete if there exists a $\mathcal{C}^{2}$ defining function $r$ of $\partial D$ defined on a neighborhood of $D=\{z \mid r(z)<0\}$ such that, with respect to a hermitian metric on $X$, at least $n-q+1$ eigenvalues of the Levi form of $-\log (-r)$ are greater than 1 on the entire $D$.

This notion originates in the work of Takeuchi [19] who showed that any proper locally pseudoconvex domain in $\mathbb{C P}^{n}$ has this property for $q=1$. Although it has already had other names, $\log \delta$-pseudoconvexity in [2], and the strong Oka condition in [11], we name it again for consistency with the terms like $q$-convexity and $q$-completeness, and Takeuchi $q$-convexity in [6].

Takeuchi 1-completeness not only implies that the domain is Stein, but also that it behaves as if it lied in complex Euclidean space:

Theorem 3.4 ([16, Theorem 1.1]). Let $D$ be a Takeuchi 1-complete domain with defining function $r$. Then $-i \partial \bar{\partial} \log (-r)$ gives a complete Kähler metric on $D$, and it follows that $-(-r)^{t_{0}}$ with sufficiently small $t_{0}>0$ becomes a strictly plurisubharmonic bounded exhaustion function on $D$, i.e., $D$ is hyperconvex.

Remark 3.5. From the viewpoint of confoliation [7, Corollary 1.1.10], we can translate any question on strong pseudoconvexity of the complement of a Leviflat real hypersurface into one on approximation of the Levi foliation by contact structures. For example, suppose a compact Levi-flat real hypersurface $M$ has a Takeuchi 1-convex complement with defining function $r$. For small positive $\varepsilon$, the level sets $\{r=-\varepsilon\}$ are diffeomorphic to $M$ and possess contact structures induced from the strictly pseudoconvex CR structures. Thus, the family of level sets defines a "uniform" contact deformation of the Levi foliation. Here "uniform" means that convergence to the foliation is exactly of the same order on the entire $M$.

\section{§3.4. Takeuchi 1-complete case}

Proposition 3.6. Let $\mathcal{D}$ be a holomorphic disc bundle over a compact Riemann surface $\Sigma$ with a uniquely determined non-holomorphic harmonic section $h$ with $\operatorname{rank}_{\mathbb{R}} d h=2$ on an open dense set. Then $\mathcal{D}$ is Takeuchi 1 -complete in its associated ruled surface $X$.

Proof. Fix a finite open covering $\left\{U_{\nu}\right\}$ of $\Sigma$ giving trivializations of $\mathcal{D}$. Set $\delta=$ $\max _{\nu} \sup _{U_{\nu}}|h|<1$ where the value of $h$ is taken with respect to the trivializing coordinate over each $U_{\nu}$. It suffices to find a defining function $r$ of $\partial \mathcal{D}$ so that the eigenvalues of the complex Hessian of $-\log (-r)$ in each trivializing coordinate 
$(z, \zeta): \pi^{-1}\left(U_{\nu}\right) \rightarrow \mathbb{C}^{2}$ are bounded from below by a positive constant, since we can easily find a hermitian metric on $X$ that is comparable to $i(d z d \bar{z}+d \zeta d \bar{\zeta})$ by the usual "partition of unity" argument.

We will find the desired $r$ in the form $r=r_{0} e^{-\psi}$ where $r_{0}$ is the defining function of $\partial \mathcal{D}$ used in [5], and $\psi: \Sigma \rightarrow \mathbb{R}$ will be determined later. Recall the original defining function

$$
r_{0}(z, \zeta):=\left|\frac{\zeta-h(z)}{1-\overline{h(z)} \zeta}\right|^{2}-1
$$

where $(z, \zeta)$ is any trivializing coordinate. It is clearly well-defined since the term inside the modulus is just a Möbius transformation that maps $h(z)$ to 0 and the remaining choices of the fiber coordinate are only up to rotations.

Take one of the trivializations, say $(z, \zeta): \pi^{-1}\left(U_{\nu}\right) \rightarrow \mathbb{C}^{2}$. The Levi form is

$$
\begin{aligned}
& i \partial \bar{\partial}(-\log (-r)) \\
& =i \partial \bar{\partial}\left(\psi-\log \left(1-|\zeta|^{2}\right)-\log \left(1-|h|^{2}\right)+2 \operatorname{Re} \log (1-\bar{h} \zeta)\right) \\
& =\left(\psi_{z \bar{z}}+\left(1-|\zeta|^{2}\right)\left(\left|h_{z}\right|^{2}+\left|h_{\bar{z}}\right|^{2}\right)+\frac{|\zeta-h|^{2}}{1-|h|^{2}}\left|h_{z}-e^{2 i \theta(z, \zeta)} \overline{h_{\bar{z}}}\right|^{2}\right) \frac{i d z \wedge d \bar{z}}{|1-\bar{h} \zeta|^{2}\left(1-|h|^{2}\right)} \\
& \quad-h_{z} \frac{i d z \wedge d \bar{\zeta}}{(1-h \bar{\zeta})^{2}}-\overline{h_{z}} \frac{i d \zeta \wedge d \bar{z}}{(1-\bar{h} \zeta)^{2}}+\frac{i d \zeta \wedge d \bar{\zeta}}{\left(1-|\zeta|^{2}\right)^{2}}
\end{aligned}
$$

where $\theta(z, \zeta):=\arg (\zeta-h) /(1-\bar{h} \zeta)$ and all the values of $h$ and $\psi$ are taken at $z$. We can check it by direct computation in three steps:

(i) Fix $z_{0} \in U$ in the trivialization. Choose a temporary trivializing coordinate $\left(z, \zeta^{\natural}\right)$ with $h^{\natural}\left(z_{0}\right)=0$.

(ii) Compute the Levi form on the fiber $\mathcal{D}_{z_{0}}$ in the $\left(z, \zeta^{\natural}\right)$ coordinate. Note that the harmonicity of $h$ yields $h_{z \bar{z}}\left(z_{0}\right)=0$.

(iii) Pull back the form to the $(z, \zeta)$ coordinate.

Now we are going to estimate the eigenvalues of the complex Hessian. The trace and determinant of the complex Hessian of $-\log (-r)$ are estimated as follows:

trace of the complex Hessian

$$
\begin{aligned}
& =\frac{1}{\left(1-|\zeta|^{2}\right)^{2}}+\frac{\psi_{z \bar{z}}+\left(1-|\zeta|^{2}\right)\left(1-|h|^{2}\right)\left(\left|h_{z}\right|^{2}+\left|h_{\bar{z}}\right|^{2}\right)+|\zeta-h|^{2}\left|h_{z}-e^{2 i \theta} \overline{h_{\bar{z}}}\right|^{2}}{|1-\bar{h} \zeta|^{2}\left(1-|h|^{2}\right)^{2}} \\
& \leq \frac{1}{\left(1-|\zeta|^{2}\right)^{2}}+\frac{\psi_{z \bar{z}}+\left(1-|\zeta|^{2}\right)\left(\left|h_{z}\right|^{2}+\left|h_{\bar{z}}\right|^{2}\right)+|\zeta-h|^{2}\left|h_{z}-e^{2 i \theta} \overline{h_{\bar{z}}}\right|^{2}}{(1-\delta)^{4}}
\end{aligned}
$$




$$
\begin{aligned}
& \leq \frac{1}{\left(1-|\zeta|^{2}\right)^{2}}+\frac{\psi_{z \bar{z}}+\left(1-|\zeta|^{2}+2|\zeta-h|^{2}\right)\left(\left|h_{z}\right|^{2}+\left|h_{\bar{z}}\right|^{2}\right)}{(1-\delta)^{4}} \\
& \leq \frac{1}{\left(1-|\zeta|^{2}\right)^{2}}+\frac{\psi_{z \bar{z}}+8\left(\left|h_{z}\right|^{2}+\left|h_{\bar{z}}\right|^{2}\right)}{(1-\delta)^{4}} \\
& \leq \frac{1}{\left(1-|\zeta|^{2}\right)^{2}}+\sup _{U} \frac{\psi_{z \bar{z}}+8\left(\left|h_{z}\right|^{2}+\left|h_{\bar{z}}\right|^{2}\right)}{(1-\delta)^{4}}=: \frac{1}{\left(1-|\zeta|^{2}\right)^{2}}+C ;
\end{aligned}
$$

determinant of the complex Hessian

$$
\begin{aligned}
= & \frac{\psi_{z \bar{z}}}{\left(1-|\zeta|^{2}\right)^{2}}+\frac{1}{\left(1-|\zeta|^{2}\right)^{2}}\left(\frac{|\zeta-h|^{2}\left|h_{z}-e^{2 i \theta} \overline{h_{\bar{z}}}\right|^{2}}{|1-\bar{h} \zeta|^{2}\left(1-|h|^{2}\right)^{2}}\right) \\
& +\frac{1}{1-|\zeta|^{2}}\left(\frac{\left|h_{\bar{z}}\right|^{2}}{|1-\bar{h} \zeta|^{2}\left(1-|h|^{2}\right)}+\frac{|\zeta-h|^{2}\left|h_{z}\right|^{2}}{|1-\bar{h} \zeta|^{4}\left(1-|h|^{2}\right)}\right) \\
\geq & \frac{\psi_{z \bar{z}}}{\left(1-|\zeta|^{2}\right)^{2}}+\frac{|\zeta-h|^{2}\left|h_{z}-e^{2 i \theta} \overline{h_{\bar{z}}}\right|^{2}}{4\left(1-|\zeta|^{2}\right)^{2}}+\frac{\left|h_{\bar{z}}\right|^{2}}{4\left(1-|\zeta|^{2}\right)}+\frac{|\zeta-h|^{2}\left|h_{z}\right|^{2}}{16\left(1-|\zeta|^{2}\right)} \\
\geq & \frac{\psi_{z \bar{z}}}{\left(1-|\zeta|^{2}\right)^{2}}+\frac{|\zeta-h|^{2}\left(\left|h_{z}\right|-\left|h_{\bar{z}}\right|\right)^{2}+\left(1-|\zeta|^{2}\right)\left|h_{\bar{z}}\right|^{2}}{4\left(1-|\zeta|^{2}\right)^{2}} \\
\geq & \frac{\psi_{z \bar{z}}}{\left(1-|\zeta|^{2}\right)^{2}}+\frac{\left(|\zeta-h|^{2}+1-|\zeta|^{2}\right) \min \left\{\left(\left|h_{z}\right|-\left|h_{\bar{z}}\right|\right)^{2},\left|h_{\bar{z}}\right|^{2}\right\}}{4\left(1-|\zeta|^{2}\right)^{2}} \\
\geq & \frac{\psi_{z \bar{z}}}{\left(1-|\zeta|^{2}\right)^{2}}+\frac{(1-\delta)^{2} \min \left\{\left(\left|h_{z}\right|-\left|h_{\bar{z}}\right|\right)^{2},\left|h_{\bar{z}}\right|^{2}\right\}}{4\left(1-|\zeta|^{2}\right)^{2}}
\end{aligned}
$$

We will set $\psi$ to have sufficiently small range so that the trace is positive, in which situation the smaller eigenvalue $\lambda$ of the complex Hessian of $-\log (-r)$ satisfies

$$
\begin{aligned}
\lambda & =\frac{\text { trace }}{2}-\sqrt{\frac{\text { trace }}{2}-\operatorname{det}} \geq \frac{\operatorname{det}}{\operatorname{trace}} \\
& \geq \frac{1}{1+C}\left(\psi_{z \bar{z}}+\frac{(1-\delta)^{2}}{4} \min \left\{\left|h_{\bar{z}}\right|^{2},\left(\left|h_{z}\right|-\left|h_{\bar{z}}\right|\right)^{2}\right\}\right) .
\end{aligned}
$$

Note that this estimate does not depend on $\zeta$, and is sharp in the sense that the smaller eigenvalue of the complex Hessian of $-\log \left(-r_{0}\right)$, which corresponds to the second term in the estimate, actually equals 0 at $(z, 0)$ if $h_{\bar{z}}(z)=0$ and tends to 0 near some points of $\partial \mathcal{D}_{z}$ if $\left|h_{z}(z)\right|=\left|h_{\bar{z}}(z)\right|$, which facts can be deduced from the explicit formula for the Levi form. This situation leads us to modify $r_{0}$ with $\psi$ strictly subharmonic on such locus in $\Sigma$.

From Lemma 3.7 below and the assumption on the rank of $d h$, we can find a non-empty relatively compact set $V \subset \Sigma$ on which both $\left|h_{\bar{z}}\right|$ and $\left|h_{z}\right|-\left|h_{\bar{z}}\right|$ never vanish. Removing a relatively compact $W \subset V$ from $\Sigma$, we obtain an open Riemann surface $\Sigma \backslash \bar{W}$, which carries a strictly subharmonic exhaustion function $\psi_{0}$. We extend $\psi_{0} \mid \Sigma \backslash V$ to $\psi_{1}$ on $\Sigma$ so that it vanishes on $W$. We take $0<c \ll 1$ 
for $\psi:=c \psi_{1}$ to satisfy, in all the trivializing coordinates, $\psi_{z \bar{z}}(1-\delta)^{-4}>-1$, and

$$
\psi_{z \bar{z}}+\frac{(1-\delta)^{2}}{4} \min \left\{\left|h_{\bar{z}}\right|^{2},\left(\left|h_{z}\right|-\left|h_{\bar{z}}\right|\right)^{2}\right\}>0 \quad \text { on } V .
$$

Using this $\psi$, we have obtained the desired defining function $r$.

Lemma 3.7. Let $\mathcal{D}, \Sigma$, and $h$ be as in Proposition 3.6. Then the zero set of $h_{\bar{z}}$ is finite.

Proof. We have the well-defined forms $\left|h_{z}\right|\left(1-|h|^{2}\right)^{-1}|d z|,\left|h_{\bar{z}}\right|\left(1-|h|^{2}\right)^{-1}|d z|$ and $\operatorname{Hopf}(h):=h_{z} \overline{h_{\bar{z}}}\left(1-|h|^{2}\right)^{-2} d z^{2}$ on $\Sigma$. The harmonicity of $h$ is equivalent to holomorphicity of $\operatorname{Hopf}(h)$, whose zero set consists of 4 (genus of $\Sigma$ ) -4 points. (Note that the assumption implies that $\pi_{1}(\Sigma)$ is non-abelian, thus the genus of $\Sigma$ is $>1$.) Therefore the zero set of $h_{\bar{z}}$ is also finite.

Question 1. What about cases (iii) and (iv)? We know an example in which $\mathcal{D}_{\rho}$ is Stein but not Takeuchi 1-complete ([16, Theorem 1.2]).

\section{$\S 4$. Bochner-Hartogs type extension theorem}

We will state a Bochner-Hartogs type extension theorem for CR sections of finite regularity, which can be obtained by procedures established in [14], [2] and [3]. Here we give a simple proof for the reader's convenience.

Theorem 4.1. Let $X$ be a connected compact complex manifold of dimension $n \geq 2, L$ a holomorphic line bundle over $X$, and $M a \mathcal{C}^{\infty}$ compact Levi-flat real hypersurface of $X$ which splits $X$ into two Takeuchi 1-complete domains, $D \sqcup D^{\prime}$. Then there exists $\kappa \in \mathbb{N}$ such that any $\mathcal{C}^{\kappa} C R$ section of $L \mid M$ extends to a holomorphic section of $L$.

Proof. We set

$$
N_{0}:=\min \left\{\begin{array}{l|l}
N \in \mathbb{N} & \begin{array}{l}
i \Theta_{h_{0}}-N i \partial \bar{\partial}(-\log (-r))<0 \text { on } D, \\
i \Theta_{h_{0}}-N i \partial \bar{\partial}\left(-\log \left(-r^{\prime}\right)\right)<0 \text { on } D^{\prime} \\
h_{0}: \text { hermitian metric of } L, \\
r\left(\text { resp. } r^{\prime}\right) \text { : defining function of } M \\
\text { which makes } D\left(\text { resp. } D^{\prime}\right) \text { Takeuchi 1-complete }
\end{array}
\end{array}\right\} .
$$

The assumption yields $N_{0}<\infty$. Put $\kappa:=\left\lceil n+1+N_{0} / 2\right\rceil(\geq 4)$. Take $h_{0}, r$, and $r^{\prime}$ to attain the minimum, and fix an arbitrary hermitian metric $g_{0}$ of $X$.

We denote by $\langle\cdot, \cdot\rangle_{g_{0}, h_{0}}$ (resp. $|\cdot|_{g_{0}, h_{0}}$ ) the fiber metric (resp. norm) of $L \otimes$ $\wedge \mathbb{C} T X^{*}$ determined by $g_{0}$ and $h_{0}$, and write $d \operatorname{vol}_{g_{0}}$ for the volume form on $X$ 
determined by $g_{0}$. Integration with respect to these metrics is denoted by

$$
\langle\langle\omega, \eta\rangle\rangle_{g_{0}, h_{0}, D}:=\int_{D}\langle\omega, \eta\rangle_{g_{0}, h_{0}} d \operatorname{vol}_{g_{0}}
$$

and we write $\|\omega\|_{g_{0}, h_{0}, D}^{2}:=\langle\omega, \omega\rangle_{g_{0}, h_{0}, D}$. We also use the following notation for function spaces:

- $\mathcal{C}_{(p, q)}^{\kappa}(X, L)$ : the space of $L$-valued $\mathcal{C}^{\kappa}(p, q)$-forms over $X$.

- $\mathcal{C}_{0,(p, q)}^{\kappa}(D, L)$ : the space of $L$-valued compactly supported $\mathcal{C}^{\kappa}(p, q)$-forms over $D$.

- $L_{(p, q)}^{2}\left(D, L ; g_{0}, h_{0}\right)$ : the space of $L$-valued measurable $(p, q)$-forms over $D$ whose $\|\cdot\|_{g_{0}, h_{0}, D}$ norm is finite.

We will omit the subscript $(p, q)$ when $(p, q)=(0,0)$.

The proof is divided into three lemmas.

Lemma 4.2. Let $s$ be a $\mathcal{C}^{\kappa} C R$ section of $L \mid M$. Then we can extend $s$ to $\tilde{s} \in$ $\mathcal{C}^{2}(X, L)$ so that

$$
|\bar{\partial} \tilde{s}|_{0}:=|\bar{\partial} \tilde{s}|_{g_{0}, h_{0}}=O\left(r^{\kappa-2}\right) \quad \text { along } M,
$$

where $r$ is any $\mathcal{C}^{\infty}$ defining function of $M$.

Proof. First, we extend $s$ to a $\mathcal{C}^{\kappa}$ section of $L$, still denoted by $s$, using a $\mathcal{C}^{\infty}$ collaring $M \times(-\epsilon, \epsilon) \rightarrow X$ of $M$ and a transversal cut-off function with sufficiently small support. Since $s \mid M$ is $\mathrm{CR}$, we can find a $\mathcal{C}^{\kappa-1}$ section of $L \mid M$, say $\alpha_{1}$, such that $\bar{\partial} s=\alpha_{1} \bar{\partial} r$ on $M$. We extend $\alpha_{1}$ to a $\mathcal{C}^{\kappa-1}$ section of $L$. Put $s_{1}:=s-\alpha_{1} r$. Then $\left|\bar{\partial} s_{1}\right|_{0}=\left|\left(\bar{\partial} s-\alpha_{1} \bar{\partial} r\right)-\bar{\partial} \alpha_{1} r\right|_{0}=O(r)$ because $\bar{\partial} s_{1}$ vanishes on $M$ and is of class $\mathcal{C}^{\kappa-2}$

Suppose we have inductively constructed a $\mathcal{C}^{\kappa-\ell}$ extension $s_{\ell}$ of $s$ with $s_{\ell}=$ $s-\alpha_{1} r-\alpha_{2} r^{2} / 2-\cdots-\alpha_{\ell} r^{\ell} / \ell$ and $\left|\bar{\partial} s_{\ell}\right|_{0}=O\left(r^{\ell}\right)$. Write $\bar{\partial} s_{\ell}=\beta_{\ell} r^{\ell}$ with $\beta_{\ell} \in$ $\mathcal{C}_{(0,1)}^{\kappa-(\ell+1)}(X, L)$. We obtain $0=\bar{\partial}^{2} s_{\ell}=\bar{\partial} \beta_{\ell} r+\bar{\partial} r \wedge \beta_{\ell}$. Thus, we can find $\alpha_{\ell+1} \in$ $\mathcal{C}^{\kappa-(\ell+1)}(X, L)$ such that $\beta_{\ell}=\alpha_{\ell+1} \bar{\partial} r$ on $M$. Putting $s_{\ell+1}:=s_{\ell}-\alpha_{\ell+1} r^{\ell+1} /(\ell+1)$ gives $\left|\bar{\partial} s_{\ell+1}\right|_{0}=\left|\left(\beta_{\ell}-\alpha_{\ell+1} \bar{\partial} r\right) r^{\ell}-\bar{\partial} \alpha_{\ell+1} r^{\ell+1}\right|_{0}=O\left(r^{\ell+1}\right)$ while $\beta_{\ell}-\alpha_{\ell+1} r^{\ell}$ is differentiable, which holds if $\kappa-(\ell+1) \geq 1$.

Letting $\tilde{s}:=s_{\kappa-2}$ completes the proof.

We will perform a correction to $\tilde{s}$ to obtain the desired holomorphic extension. Once we solve the $\bar{\partial}$-equation $\bar{\partial} u=\bar{\partial} \tilde{s}$ on $X$ in the distribution sense with the condition $u \mid M=0$, we obtain the desired extension $\tilde{s}-u$ since holomorphic functions are characterized as weak solutions of the Cauchy-Riemann equation. 


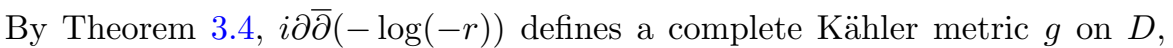
which blows up with rate $O\left(r^{-2}\right)$ along $M$. Consider the hermitian metric $h=$ $h_{0} r^{-N_{0}}$ on $L$. The condition (4.1) on $\tilde{s}$ implies

$$
\begin{aligned}
\|\bar{\partial} \tilde{s}\|_{g, h}^{2} & :=\|\bar{\partial} \tilde{s}\|_{g, h, D}^{2} \\
& =\int_{D}|\bar{\partial} \tilde{s}|_{g, h}^{2} d \operatorname{vol}_{g}=\int_{D} O\left(r^{2(\kappa-2)}\right) O\left(r^{2}\right) O\left(r^{-N_{0}}\right) O\left(r^{-2 n}\right)<\infty
\end{aligned}
$$

i.e., $\bar{\partial} \tilde{s} \in L_{(0,1)}^{2}(D, L ; g, h)$. We can solve $\bar{\partial} u=\bar{\partial} \tilde{s}$ on $D$ thanks to the following $L^{2}$ cohomology vanishing theorem.

Lemma 4.3. For any $v \in L_{(0,1)}^{2}(D, L ; g, h)$ with $\bar{\partial} v=0$, there exists a solution $u \in L^{2}(D, L ; g, h)$ of $\bar{\partial} u=v$ in the sense that there exists a sequence $u_{n} \in$ $\mathcal{C}_{0}^{\infty}(D, L)$ such that $u_{n} \rightarrow u$ in $L^{2}(D, L ; g, h)$ and $\bar{\partial} u_{n} \rightarrow v$ in $L_{(0,1)}^{2}(D, L ; g, h)$.

Proof. By the standard $L^{2}$ method of Andreotti-Vesentini, the conclusion follows from the estimate

$$
\|\bar{\partial} u\|_{g, h}^{2}+\left\|\bar{\partial}_{g, h}^{*} u\right\|_{g, h}^{2} \gtrsim\|u\|_{g, h}^{2}
$$

for $u \in C_{0,(0,1)}^{\infty}(D, L)$. Here we denote by $\bar{\partial}_{g, h}^{*}$ the formal adjoint of the operator $\bar{\partial}: L^{2}(D, L ; g, h) \rightarrow L_{(0,1)}^{2}(D, L ; g, h)$. Note that we have used the completeness of $g$ to obtain the solution not only in the sense of distributions but also in the sense above.

By the Nakano inequality, we achieve the estimate as follows:

$$
\begin{aligned}
\|\bar{\partial} u\|_{g, h}^{2}+\left\|\bar{\partial}_{g, h}^{*} u\right\|_{g, h}^{2} & \gtrsim\left\langle\left\langle\left[i \Theta_{h}, \Lambda\right] u, u\right\rangle_{g, h}=-\left\langle\left\langle i \Theta_{h} u, L u\right\rangle\right\rangle_{g, h}\right. \\
& \gtrsim-\min \left\{\begin{array}{l}
\text { sum of the }(n-1) \text { eigenvalues of } i \Theta_{h} \\
\text { with respect to } g
\end{array}\right\}\|u\|_{g, h}^{2} .
\end{aligned}
$$

The eigenvalues of $i \Theta_{h}$ with respect to $g$ tend to $-N_{0}$ near $M$. It follows that RHS $\gtrsim\|u\|_{g, h}^{2}$.

Performing the same procedure on $D^{\prime}$, we obtain a section $u$ of $L \mid D \sqcup D^{\prime}$ with $\bar{\partial} u=\bar{\partial} \tilde{s}$ on $D \sqcup D^{\prime}$ in the sense above. Consider the zero extension of $u$ on $X$, still denoted by $u$. The following lemma completes the proof of Theorem 4.1.

Lemma 4.4. $\bar{\partial} u=\bar{\partial} \tilde{s}$ on $X$ in the sense of distribution.

Proof. Let $u_{n} \in \mathcal{C}_{0}^{\infty}\left(D \sqcup D^{\prime}, L\right) \subset \mathcal{C}^{\infty}(X, L)$ be the approximation of $u$ found in Lemma 4.3. Since $L^{2}\left(D, L ; g_{0}, h_{0}\right) \hookrightarrow L^{2}(D, L ; g, h)$ is continuous, we have $u_{n} \rightarrow u$ in $L^{2}\left(D \sqcup D^{\prime}, L ; g_{0}, h_{0}\right) \simeq L^{2}\left(X, L ; g_{0}, h_{0}\right)$. 
Take a test function $\phi \in \mathcal{C}^{\infty}(X, L)$. Denote by $\bar{\partial}_{0}^{*}$ the formal adjoint of the operator $\bar{\partial}: L^{2}\left(X, L ; g_{0}, h_{0}\right) \rightarrow L_{(0,1)}^{2}\left(X, L ; g_{0}, h_{0}\right)$. Then

$$
\begin{aligned}
\langle\langle\bar{\partial} u-\bar{\partial} \tilde{s}, \phi\rangle\rangle_{g_{0}, h_{0}, X} & =\left\langle\left\langle u, \bar{\partial}_{0}^{*} \phi\right\rangle\right\rangle_{g_{0}, h_{0}, X}-\langle\langle\bar{\partial} \tilde{s}, \phi\rangle\rangle_{g_{0}, h_{0}, X} \\
& =\lim _{n \rightarrow \infty}\left\langle\left\langle u_{n}, \bar{\partial}_{0}^{*} \phi\right\rangle\right\rangle_{g_{0}, h_{0}, X}-\langle\langle\bar{\partial} \tilde{s}, \phi\rangle\rangle_{g_{0}, h_{0}, X} \\
& =\lim _{n \rightarrow \infty}\left\langle\left\langle u_{n}, \bar{\partial}_{0}^{*} \phi\right\rangle\right\rangle_{g_{0}, h_{0}, D \sqcup D^{\prime}}-\langle\langle\bar{\partial} \tilde{s}, \phi\rangle\rangle_{g_{0}, h_{0}, D \sqcup D^{\prime}} \\
& =\lim _{n \rightarrow \infty}\left\langle\left\langle\bar{\partial} u_{n}-\bar{\partial} \tilde{s}, \phi\right\rangle\right\rangle_{g_{0}, h_{0}, D \sqcup D^{\prime}}=0 .
\end{aligned}
$$

Corollary 4.5. Suppose $X, L, M$, and $\kappa$ are as in Theorem 4.1. Then all $\mathcal{C}^{\kappa} C R$ sections of $L \mid M$ are automatically of class $\mathcal{C}^{\infty}$, and they form a finite-dimensional vector space.

We will use the following form of Theorem 4.1 in the proof of the Main Theorem.

Corollary 4.6. Suppose $X, L$ and $M$ are as in Theorem 4.1. Then any $\mathcal{C}^{\infty} C R$ section of $L \mid M$ extends to a holomorphic section of $L$.

\section{$\S 5$. Proof of the Main Theorem}

From Proposition 3.6, $\mathcal{D}$ is Takeuchi 1-complete. The harmonic section of $X \backslash \overline{\mathcal{D}}$ is obtained by conjugating the harmonic section of $\mathcal{D}$. Thus, $X \backslash \overline{\mathcal{D}}$ is also Takeuchi 1-complete. Hence, Corollary 4.6 implies that for any $n \geq 1$, all $\mathcal{C}^{\infty} \mathrm{CR}$ sections of $\left(\pi^{*} L \mid M\right)^{\otimes n}$ extend to holomorphic sections of $\left(\pi^{*} L\right)^{\otimes n}$.

On the other hand, $\pi^{*}: H^{0}\left(\Sigma, L^{\otimes n}\right) \rightarrow H^{0}\left(X,\left(\pi^{*} L\right)^{\otimes n}\right)$ gives an isomorphism. We can give a trivializing cover of $\left(\pi^{*} L\right)^{\otimes n}$ by pulling back that of $L$, and the sections should be constant along any fiber $\pi^{-1}(p) \simeq \mathbb{C P}^{1}$ in these trivializations. Hence it is impossible for the sections in $H^{0}\left(X,\left(\pi^{*} L\right)^{\otimes n}\right)$ to separate points in the same fiber for any $n$. Therefore, we cannot make a projective embedding by any ratio of those sections.

We conclude this paper with further questions.

Question 2. Can one prove the Main Theorem intrinsically, i.e., without considering the natural Stein filling?

Question 3. Let $M$ be a compact Levi-flat CR manifold, and $L$ a CR line bundle over $M$. We define the threshold regularity $\kappa(M, L)$ to be the minimal $\kappa \in \mathbb{N} \cup\{\infty\}$, if exists, so that the $\mathcal{C}^{\kappa} \mathrm{CR}$ sections of $L$ form a finite-dimensional vector space. In the situation illustrated in the Main Theorem, the proof of Theorem 4.1 indicates 
that $\kappa\left(M,\left(\pi^{*} L \mid M\right)^{\otimes n}\right)$ is well-defined and $\kappa\left(M,\left(\pi^{*} L \mid M\right)^{\otimes n}\right)=O(n)$ as $n \rightarrow \infty$. On the other hand, Ohsawa-Sibony's projective embedding theorem implies that $\kappa\left(M,\left(\pi^{*} L \mid M\right)^{\otimes n}\right) \rightarrow \infty$ as $n \rightarrow \infty$. Can we read off any dynamical property of the Levi foliation from the asymptotic behavior of $\kappa\left(M,\left(\pi^{*} L \mid M\right)^{\otimes n}\right)$ ?

\section{Acknowledgments}

The author would like to express his profound gratitude to his advisor T. Ohsawa. He is grateful to J. Brinkschulte for pointing out a mistake in a previous version of this article, and also to B.-Y. Chen for useful comments on hyperconvexity. He thanks R. Kobayashi and K. Matsumoto for helpful comments which improved the presentation of this paper. Part of this work was done during "École d'été 2012: Feuilletages, courbes pseudoholomorphes, applications" at Institut Fourier, and "Nagoya-Tongji joint workshop on Bergman kernel" at Tongji University. He is grateful to both institutes for their hospitality and support.

\section{References}

[1] D. E. Barrett, Global convexity properties of some families of three-dimensional compact Levi-flat hypersurfaces, Trans. Amer. Math. Soc. 332 (1992), 459-474. Zbl 0761.32010 MR 1055805

[2] J. Brinkschulte, The $\bar{\partial}$-problem with support conditions on some weakly pseudoconvex domains, Ark. Mat. 42 (2004), 259-282. Zbl 1078.32023 MR 2101387

[3] D. Chakrabarti and M.-C. Shaw, $L^{2}$ Serre duality on domains in complex manifolds and applications, Trans. Amer. Math. Soc. 364 (2012), 3529-3554. Zbl 1276.32008 MR 2901223

[4] B. Deroin, Laminations dans les espaces projectifs complexes, J. Inst. Math. Jussieu 7 (2008), 67-91. Zbl 1153.32004 MR 2398147

[5] K. Diederich and T. Ohsawa, Harmonic mappings and disc bundles over compact Kähler manifolds, Publ. RIMS Kyoto Univ. 21 (1985), 819-833. Zbl 0601.32023 MR 0817167

[6] On the displacement rigidity of Levi flat hypersurfaces - the case of boundaries of disc bundles over compact Riemann surfaces, Publ. RIMS Kyoto Univ. 43 (2007), 171-180. Zbl 1141.53040 MR 2319541

[7] Y. M. Eliashberg and W. P. Thurston, Confoliations, Univ. Lecture Ser. 13, Amer. Math. Soc., Providence, RI, 1998. MR 1483314

[8] J. E. Fornæss and E. F. Wold, Solving $\bar{\partial}_{b}$ on hyperbolic laminations, arXiv:1108.2286 [math.CV] (2011)

[9] É. Ghys, Laminations par surfaces de Riemann, in Dynamique et géométrie complexes (Lyon, 1997), Panor. Synthèses 8, Soc. Math. France, Paris, 1999, ix, xi, 49-95 Zbl 1018.37028 MR 1760843

[10] M. Gromov, Topological invariants of dynamical systems and spaces of holomorphic maps. I, Math. Phys. Anal. Geom. 2 (1999), 323-415. Zbl 1160.37322 MR 1742309

[11] P. S. Harrington and M.-C. Shaw, The strong Oka's lemma, bounded plurisubharmonic functions and the $\bar{\partial}$-Neumann problem, Asian J. Math. 11 (2007), 127-139. Zbl 1130.32016 MR 2304586 
[12] T. Inaba, On the nonexistence of CR functions on Levi-flat CR manifolds, Collect. Math. 43 (1992), 83-87. Zbl 0783.32006 MR 1214226

[13] D. Martínez Torres, Generic linear systems for projective CR manifolds, Differential Geom. Appl. 29 (2011), 348-360. Zbl 1226.32019 MR 2795843

[14] T. Ohsawa, Pseudoconvex domains in $\mathbf{P}^{n}$ : a question on the 1-convex boundary points, in Analysis and geometry in several complex variables (Katata, 1997), Trends Math., Birkhäuser Boston, Boston, MA, 1999, 239-252. Zbl 0971.32007 MR 1699848

[15] On projectively embeddable complex-foliated structures, Publ. RIMS Kyoto Univ. 48 (2012), 735-747. Zbl 1262.32027 MR 2973399

[16] T. Ohsawa and N. Sibony, Bounded p.s.h. functions and pseudoconvexity in Kähler manifold, Nagoya Math. J. 149 (1998), 1-8. Zbl 0911.32027 MR 1619572

[17] Kähler identity on Levi flat manifolds and application to the embedding, Nagoya Math. J. 158 (2000), 87-93. Zbl 0976.32021 MR 1766573

[18] J. H. Sampson, Some properties and applications of harmonic mappings, Ann. Sci. École Norm. Sup. (4) 11 (1978), 211-228. Z Zbl 0392.31009 MR 0510549

[19] A. Takeuchi, Domaines pseudoconvexes infinis et la métrique riemannienne dans un espace projectif, J. Math. Soc. Japan 16 (1964), 159-181. Zbl 0141.08804 MR 0173789 